\title{
Leucine-Rich Alpha-2 Glycoprotein May Be Predictive of the Adalimumab Trough Level and Antidrug Antibody Development for Patients with Inflammatory Bowel Disease: A Sub-Analysis of the PLANET Study
}

\author{
Shunichi Yanai ${ }^{a}$ Shinichiro Shinzaki ${ }^{b}$ Katsuyoshi Matsuokac Shinta Mizuno ${ }^{d}$

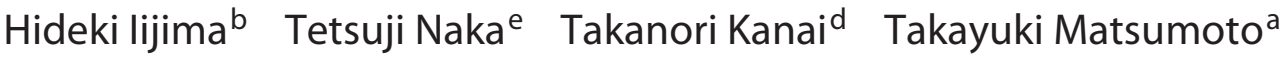 \\ aDivision of Gastroenterology, Department of Internal Medicine, Iwate Medical University, Iwate, Japan; \\ ${ }^{b}$ Department of Gastroenterology and Hepatology, Osaka University Graduate School of Medicine, Osaka, Japan; \\ 'Division of Gastroenterology and Hepatology, Department of Internal medicine, Toho University Sakura Medical \\ Center, Chiba, Japan; ${ }^{d}$ Division of Gastroenterology and Hepatology, Department of Internal Medicine, Keio \\ University, Tokyo, Japan; 'Department of Clinical Immunology, Kochi University, Kochi, Japan
}

\section{Keywords}

Crohn's disease - Ulcerative colitis · Leucine-rich alpha-2

glycoprotein · Adalimumab · Antidrug antibodies

\begin{abstract}
Introduction: The aim of this study was to examine whether biomarkers are predictive of the adalimumab (ADA) trough level and antidrug antibody development in patients with Crohn's disease (CD) and ulcerative colitis (UC). Methods: Using data obtained in a prospective, multicenter, observational study (PLANET), we assessed serial changes in a novel biomarker - leucine-rich alpha-2 glycoprotein (LRG) - during ADA treatment for patients with active $C D$ and UC. We measured serum LRG, C-reactive protein (CRP), and fecal calprotectin (fCAL) at weeks $0,12,24$, and 52. The ADA trough level and anti-ADA antibody (AAA) were also measured at weeks 12 and 52. Correlations between the ADA trough level, AAA, and biomarkers were examined. Results: In all, 34
\end{abstract}

karger@karger.com www.karger.com/dig

Karger $\stackrel{\text { ' }}{5}$

GOPEN ACCESS
(C) 2021 The Author(s)

Published by S. Karger AG, Basel

This is an Open Access article licensed under the Creative Commons Attribution-NonCommercial-4.0 International License (CC BY-NC) (http://www.karger.com/Services/OpenAccessLicense), applicable to the online version of the article only. Usage and distribution for commercial purposes requires written permission. patients with $C D$ and 47 patients with UC were enrolled. The ADA trough level at week 12 or at the time of ADA withdrawal was $8.5 \pm 3.9$ in the AAA-negative group $(n=70)$ and $2.9 \pm$ $2.7 \mu \mathrm{g} / \mathrm{mL}$ in the AAA-positive group $(n=8)(p<0.0001)$. The ADA trough level at week 12 or at the time of ADA withdrawal was associated with pretreatment LRG ( $p=0.0437$ and $r=-0.23)$. Conclusion: $L R G$, rather than CRP or fCAL, may be a marker for predicting the trough level of ADA for patients with $C D$ and $U C$ treated with ADA.

$$
\begin{aligned}
& \text { (c) } 2021 \text { The Author(s) } \\
& \text { Published by S. Karger AG, Basel }
\end{aligned}
$$

\section{Introduction}

Since the introduction of infliximab, antitumor necrosis factor (TNF) antibodies have become a mainstay for the treatment of inflammatory bowel disease (IBD), including Crohn's disease (CD) and ulcerative colitis (UC) [1]. Recently, biologic agents targeting other molecules, 
such as ustekinumab and vedolizumab, have been shown to be efficacious for IBD, especially for patients who do not respond to anti-TNF antibodies [2,3]. The efficacy of those biologics has impacted the strategies and goals for the management of IBD, and it has recently become widely recognized that endoscopic and histologic healing is the main goal of treatment [4].

While the biologics are efficacious in IBD, there are concerns that should be considered, including infectious and noninfectious adverse events and loss of efficacy during a prolonged period of treatment. It has become evident that loss of response to biologics is closely associated with the trough level of and antibody development against these agents [5-8]. In order to prevent loss of response to anti-TNF antibodies, therapeutic drug monitoring (TDM), including the trough level and antibody titers, has been suggested [5-13]. In clinical practice, however, measurements of trough levels and antibody levels are time-consuming and expensive.

We have recently undertaken a prospective, observational study that evaluated serum leucine-rich alpha-2 glycoprotein (LRG) as a biomarker for disease activity in IBD (PLANET study) [14]. We found that LRG is a candidate that is equal or possibly superior to C-reactive protein (CRP) and fecal calprotectin (fCAL) for the assessment of disease activity in IBD treated with adalimumab (ADA). In the present investigation, we elucidated whether serum LRG is associated with TDM parameters at week 12 after the start of induction therapy with ADA.

\section{Materials and Methods}

\section{Patients}

This was a multicenter, prospective, observational study conducted between January 2016 and March 2018 at 20 institutions in Japan. This study was registered in the University Hospital Medical Information Network (registration \#000019958), and it was conducted according to the Declaration of Helsinki and Ethical Guidelines for Medical and Health Research Involving Human Subjects provided by the Japan Ministry of Health, Labour and Welfare. All patients provided written informed consent.

Patients were recruited at the time of starting ADA and evaluated for 52 weeks or until drug withdrawal. The inclusion criteria for patients with CD were as follows: (1) age 15 years or older; (2) moderately-to-severely active CD defined with a CD Activity Index (CDAI) score of 220 or more; and (3) insufficient response to conventional therapies such as corticosteroids and immunomodulators. CD was diagnosed on the basis of established diagnostic criteria [15]. Exclusion criteria included bowel resection within 12 weeks, patients with stoma or ileal pouch (excluding ileo-rectal anastomosis), prior exposure to ADA, history of primary nonresponse to infliximab or secondary nonresponse to infliximab at a dose of $10 \mathrm{mg} / \mathrm{kg}$, and contraindications to ADA. For inclusion,
Table 1. Demographic data and baseline clinical characteristics of patients $(n=81)$

\section{Gender}

Male, $n(\%)$

Female, $n(\%)$

Age, years, mean (range)

BMI, mean (range)

Disease

$\mathrm{CD}, n(\%)$

$\mathrm{UC}, n(\%)$

Disease activity

CDAI, mean (range)

Partial Mayo score, mean (range)

Perianal lesion (CD), $n(\%)$

Disease location (CD)

Ileitis, $n(\%)$

Colitis, $n(\%)$

Ileocolitis, $n(\%)$

Disease location (UC)

Total, $n(\%)$

Left-sided, $n(\%)$

Right-sided or segmental, $n$ (\%)

Concomitant medications

5-aminosalicylic acid, $n$ (\%)

Azathioprine

6-mercaptopurine

Corticosteroids, $n$ (\%)

Previous use of biologics, $n$ (\%)

AAA positive, $n(\%)$
$50(61.7)$

$31(38.3)$

$35.9(15-70)$

$20.2(11.7-29.7)$

$34(42)$

$47(58)$

$271(183-409)$

$5.9(4-9)$

$21(61.8)$

$3(8.8)$

$4(11.8)$

$27(79.4)$

$30(63.8)$

$16(34.0)$

$1(2.1)$

$70(86.4)$

$24(29.6)$

$5(6.1)$

$43(53.0)$

$8(9.8)$

$8(9.8)$
AAA, anti-adalimumab antibody; BMI, body mass index; CD, Crohn's disease; CDAI, Crohn's Disease Activity Index; UC, ulcerative colitis.
UC patients must have been diagnosed with UC with moderate to severe clinical activity according to a Mayo score of at least 6 [16]. UC patients were excluded if they were 14 years old or younger, underwent a proctocolectomy, had a stoma, had prior use of ADA, or experienced a primary failure to induction of infliximab or a secondary failure to $10 \mathrm{mg} / \mathrm{kg}$ of infliximab. Patients with severe infection, active tuberculosis, demyelinating disease, or chronic heart failure were also excluded.

Study Design

Study visits were scheduled at the time of the first dose of ADA (week 0) and at weeks 12, 24, and 52. Serum LRG levels, fCAL levels, partial Mayo scores, and CDAI scores were evaluated at weeks $0,12,24$, and 52 . If a patient discontinued the study before week 52 , these variables were measured at the time of study discontinuation. Serum trough levels of ADA and anti-ADA antibodies (AAA) were measured at weeks 12 and 52 or at the time of discontinuation of the study. Serum LRG levels were measured by latex turbidimetric immunoassay (Sekisui Medical, Tokyo, Japan), as has been described elsewhere [17]. CRP levels were measured at each institution. Stool samples for fCAL measurement were collected at weeks $0,12,24$, and 52 and analyzed by using an EliA ${ }^{\mathrm{TM}}$ Calprotectin kit (Thermo Fisher Diagnostics K.K., Tokyo, Japan). 
Table 2. Comparison of AAA-positive and AAA-negative patients

\begin{tabular}{|c|c|c|c|}
\hline & $\begin{array}{l}\text { AAA positive } \\
(n=8)\end{array}$ & $\begin{array}{l}\text { AAA negative } \\
(n=70)\end{array}$ & $p$ value \\
\hline Gender (male/female), $n$ & $6 / 2$ & $43 / 27$ & 0.7082 \\
\hline Age, mean $\pm \mathrm{SD}$, years & $35.1 \pm 16.1$ & $36.1 \pm 15.3$ & 0.8598 \\
\hline Duration of disease, mean $\pm S D$, years & $6.9 \pm 6.9$ & $5.9 \pm 7.1$ & 0.6892 \\
\hline $\mathrm{BMI}$, mean $\pm \mathrm{SD}, \mathrm{kg} / \mathrm{m}^{2}$ & $20.3 \pm 2.1$ & $20.3 \pm 3.5$ & 0.9407 \\
\hline \multicolumn{4}{|l|}{ Disease } \\
\hline $\mathrm{UC}, n$ & 7 & 37 & 0.1283 \\
\hline $\mathrm{CD}, n$ & 1 & 33 & \\
\hline Immunomodulator & 1 & 25 & 0.4118 \\
\hline Previous use of biologics & 3 & 5 & 0.0318 \\
\hline ADA trough (week 12 ), mean $\pm \mathrm{SD}, \mu \mathrm{g} / \mathrm{dL}$ & $2.9 \pm 2.7$ & $8.9 \pm 3.8$ & $<0.0001$ \\
\hline $\mathrm{CRP}($ week 12$)$, mean $\pm \mathrm{SD}, \mathrm{mg} / \mathrm{dL}$ & $0.45 \pm 0.93$ & $0.31 \pm 0.64$ & 0.5872 \\
\hline $\mathrm{LRG}$ (week 12 ), mean $\pm \mathrm{SD}, \mu \mathrm{g} / \mathrm{mL}$ & $16.8 \pm 6.6$ & $15.3 \pm 7.8$ & 0.6449 \\
\hline
\end{tabular}

AAA, anti-adalimumab antibody; BMI, body mass index; CD, Crohn's disease; CRP, C-reactive protein; LRG, leucine-rich alpha-2 glycoprotein; UC, ulcerative colitis.

\section{Measurement of ADA Trough and AAA}

Serum ADA trough concentrations were measured by electrochemiluminescence (ECL). Immobilized antigen solution $(50 \mu \mathrm{L})$ was added to each well of the ECL plate, and the plate was incubated at $5^{\circ} \mathrm{C}$ overnight. The fluid content was discarded, $300 \mu \mathrm{L}$ of blocking buffer was added to each well of the ECL plate, and the ECL plate was incubated at room temperature for 2-3 h. The ECL plate was washed 3 times with wash buffer. A 20-fold diluted sample $(50 \mu \mathrm{L})$ was added in duplicate to each well of the ECL plate, and the plate was incubated at room temperature for $2 \mathrm{~h}$. The ECL plate was washed 3 times with wash buffer. SULFO-TAG-labeled detection antibody solution $(50 \mu \mathrm{L})$ was added to each well of the ECL plate, and the plate was incubated at room temperature for 1 h. The ECL plate was washed 3 times with wash buffer. Read buffer $\mathrm{T}(2 \times)(150 \mu \mathrm{L})$ was added to each well of the ECL plate. The signal was measured by ECL.

A positive AAA result was determined by the results of ECL and absorption tests. From the screening assay, samples were selected that were potentially positive for the presence of AAA. The absorption rate was calculated by comparing ECL signals between samples with and without ADA. The absorption rate of individual sera from healthy individuals without drug was calculated. The mean inhibition plus 3.09 standard deviation represented the specificity cutoff point. The samples that were positive in the screening test were confirmed in the absorption test. The samples were measured with or without $\mathrm{ADA}$, and the absorption rate was calculated. Measurement samples with an absorption rate greater than $24.0 \%$ were judged as positive [18].

\section{Treatment}

ADA was administered at a dosage of $160 \mathrm{mg}$ subcutaneously at week 0 , followed by $80 \mathrm{mg}$ at week 2 , and then $40 \mathrm{mg}$ every other week. The dose could be increased to $80 \mathrm{mg}$ if response was lost. The following drugs were permitted to be used concomitantly if the dosage had been stable during the 4 weeks prior to the start of the study and was maintained until the end of the study (the dose

LRG, Adalimumab Trough Level, and Antidrug Antibodies could be reduced according to the patient's condition): 5-aminosalicylic acid, azathioprine, 6-mercaptopurine, methotrexate, oral corticosteroids, antibiotics for $\mathrm{CD}$, and enteral nutrition. Concomitant use of biologics other than $\mathrm{ADA}$, intravenous corticosteroids, rectal corticosteroids, 5-aminosalicylic acid, cyclosporine, tacrolimus, cytapheresis, and total parenteral nutrition were prohibited from the start to the end of the study.

\section{Statistical Analysis}

Continuous variables are presented as means with standard deviation. The Wilcoxon signed rank test was used to compare nonparametric paired values, and the paired $t$ test was used to compare parametric paired values. Differences between groups were analyzed by the Kruskal-Wallis test for nonparametric data and Student's $t$ test for parametric values. Categorical values are presented in numbers (\%), and differences were analyzed by the $\chi^{2}$ test or Fisher's exact test. Pearson's test was used for analyses of the relationships between ADA trough level and LRG, fCAL, CRP, and body mass index (BMI). In each statistical analysis, probabilities < 0.05 were considered statistically significant.

\section{Results}

\section{Patients}

Table 1 summarizes demographic data and baseline clinical characteristics of the study population. A total of 34 patients with CD and 47 patients with UC were enrolled for the study, among whom 34 patients with CD and 36 patients with UC completed the study protocol up to week 12 . There were 50 men and 31 women with ages ranging from 15 to 70 years (mean, 35.9 years). In 3 patients, either the ADA trough level, AAA, or both were 
Fig. 1. ADA trough levels at week 12 or withdrawal for AAA-positive and AAAnegative patients. AAA, anti-adalimumab antibody; ADA, adalimumab.
Fig. 2. Comparison of serial changes in LRG between AAA-positive and AAAnegative patients. LRG, leucine-rich alpha-2 glycoprotein; AAA, anti-adalimum$\mathrm{ab}$ antibody.
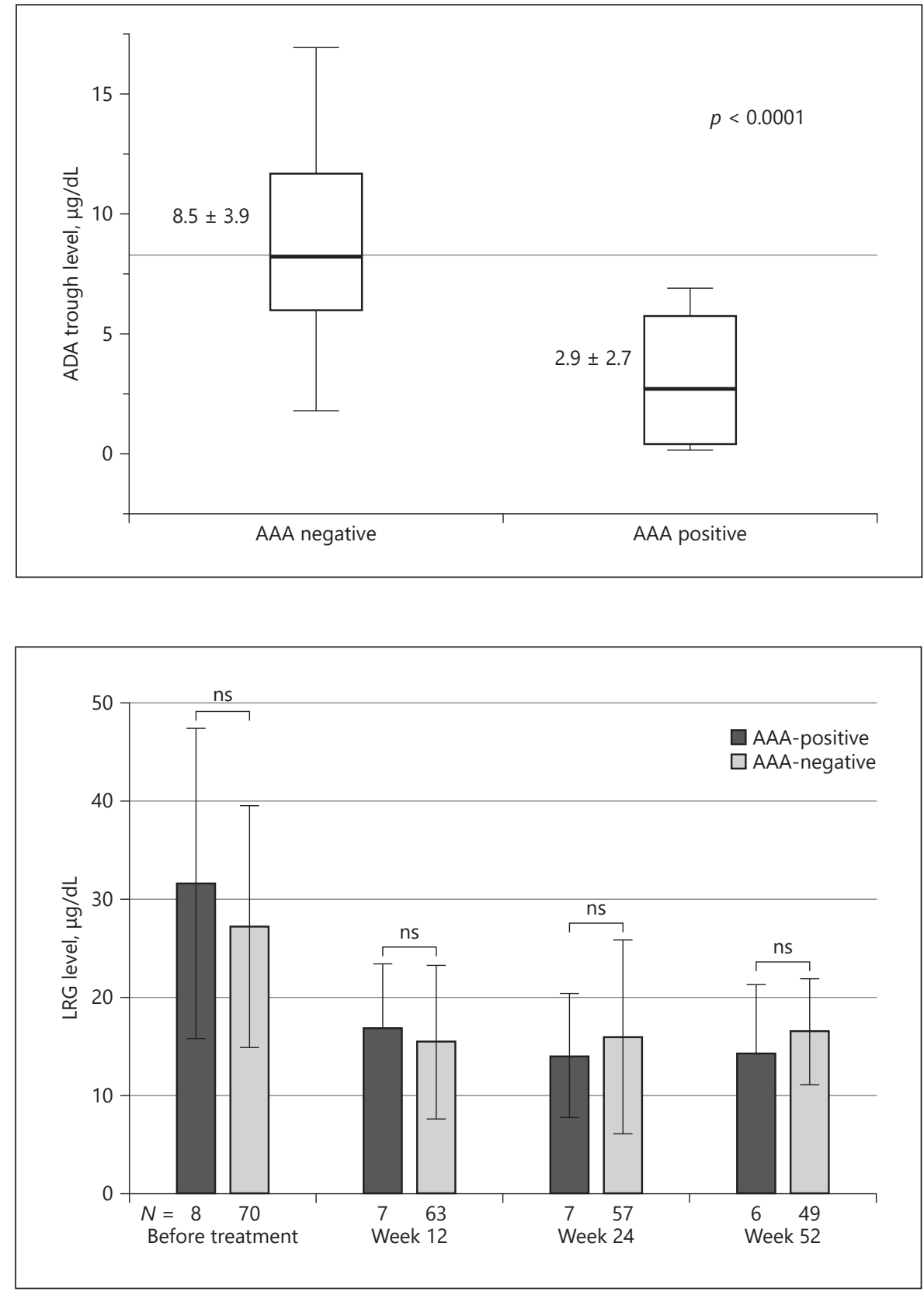

not measured at any time. The ADA trough level was measured at the time of withdrawal from the study in 8 patients. As a consequence, data from 78 patients were used for the analysis of the associations between biomarkers, ADA trough level, and AAA.

\section{Comparison of AAA-Positive and AAA-Negative \\ Patients}

Table 2 compares the clinical features between AAApositive patients and AAA-negative patients. In all, 70 pa- tients were considered AAA-negative, and 8 patients were considered AAA positive. Three patients were AAA positive at week 12 , and another 4 patients were AAA positive at week 52 . The remaining patient was AAA positive at the time of withdrawal from the study (week 25). No differences were found between the 2 groups with regard to gender, age, duration of disease, BMI, use of immunomodulatory therapy, or underlying disease. The rate of prior use of biologics was higher in the AAA-positive group than in the AAA-negative group. The ADA 


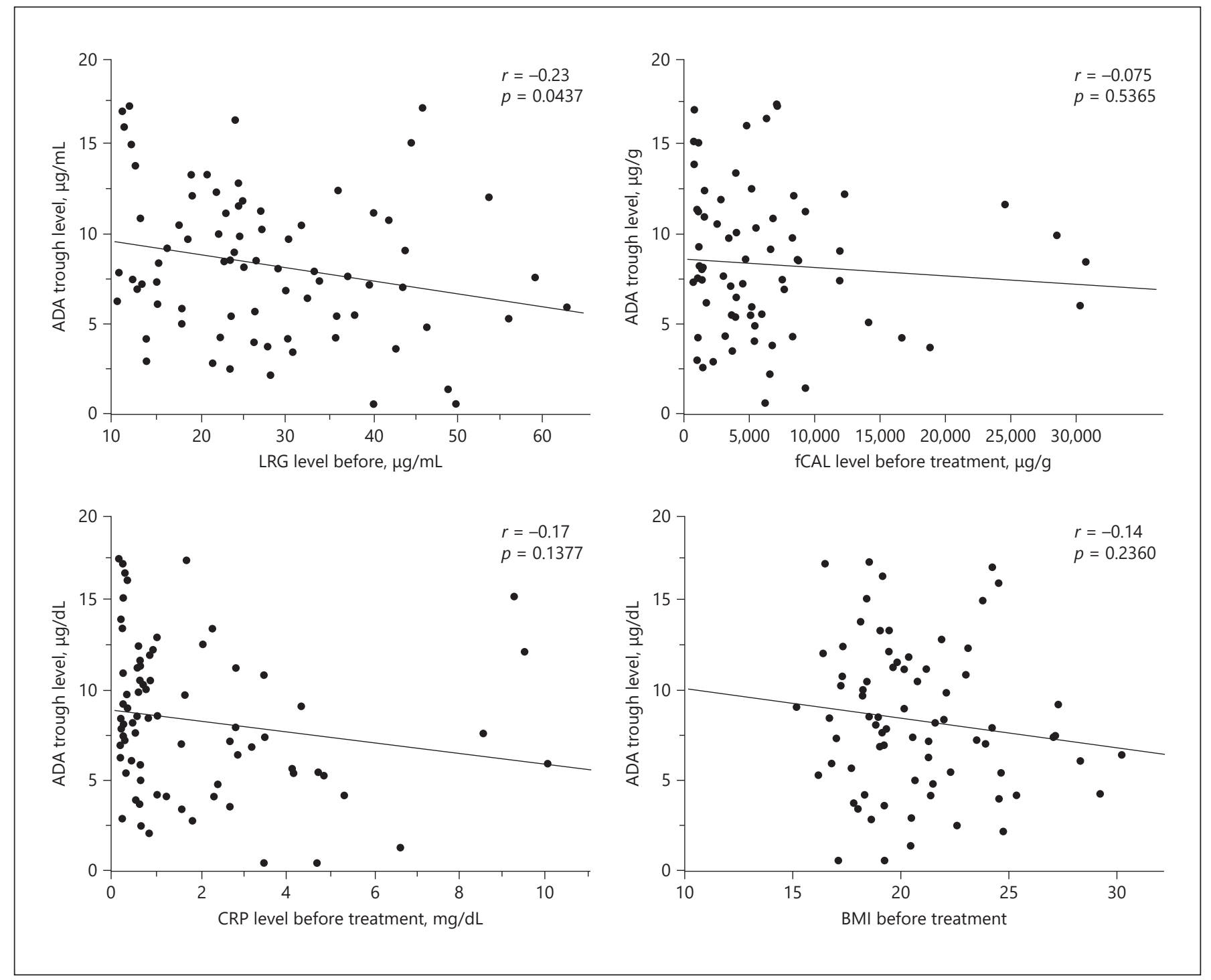

Fig. 3. Associations between the ADA trough level at week 12 or withdrawal and LRG, fCAL, CRP, and BMI before treatment. ADA, adalimumab; BMI, body mass index; CRP, C-reactive protein; fCAL, fecal calprotectin; LRG, leucine-rich alpha-2 glycoprotein.

trough level at week 12 or at the time of withdrawal was $8.5 \pm 3.9$ for the AAA-negative group and $2.9 \pm 2.7 \mu \mathrm{g} / \mathrm{mL}$ for the AAA-positive group $(p<0.0001)$ (Fig. 1). The ADA trough level at week 52 or ADA withdrawal was 11.2 \pm 5.7 in the AAA-negative group and $0.6 \pm 1.2 \mu \mathrm{g} / \mathrm{mL}$ in the AAA-positive group $(p<0.0001)$.

Figure 2 shows a comparison of serial changes in LRG between AAA-positive and AAA-negative patients. As shown in the figure, LRG decreased after the start of ADA treatment in each group. However, LRG was not different between the 2 groups at any time point.

LRG, Adalimumab Trough Level, and Antidrug Antibodies
Correlations between ADA Trough Level and $L R G$, $C R P, f C A L$, and BMI

Figure 3 presents the correlations between ADA trough level at week 12 or withdrawal and LRG, CRP, fCAL, and BMI prior to ADA treatment. There was a statistically significant correlation between the ADA trough level and pretreatment LRG ( $p=0.0437$ and $r=-0.23)$. In contrast, the ADA trough level at week 12 or withdrawal was not significantly associated with pretreatment BMI $(p=0.2360$ and $r=-0.14), \operatorname{CRP}(p=0.1377$ and $r=-0.17)$, or fCAL $(p=0.5365, r=-0.075)$. 
Fig. 4. Comparison of pretreatment LRG levels among quartiles classified by ADA trough level at week 12 or at the time of withdrawal. LRG, leucine-rich alpha-2 glycoprotein; ADA, adalimumab.

Table 3. Correlations between ADA trough level at week 52 and LRG, CRP, fCAL, and BMI $(n=55)$

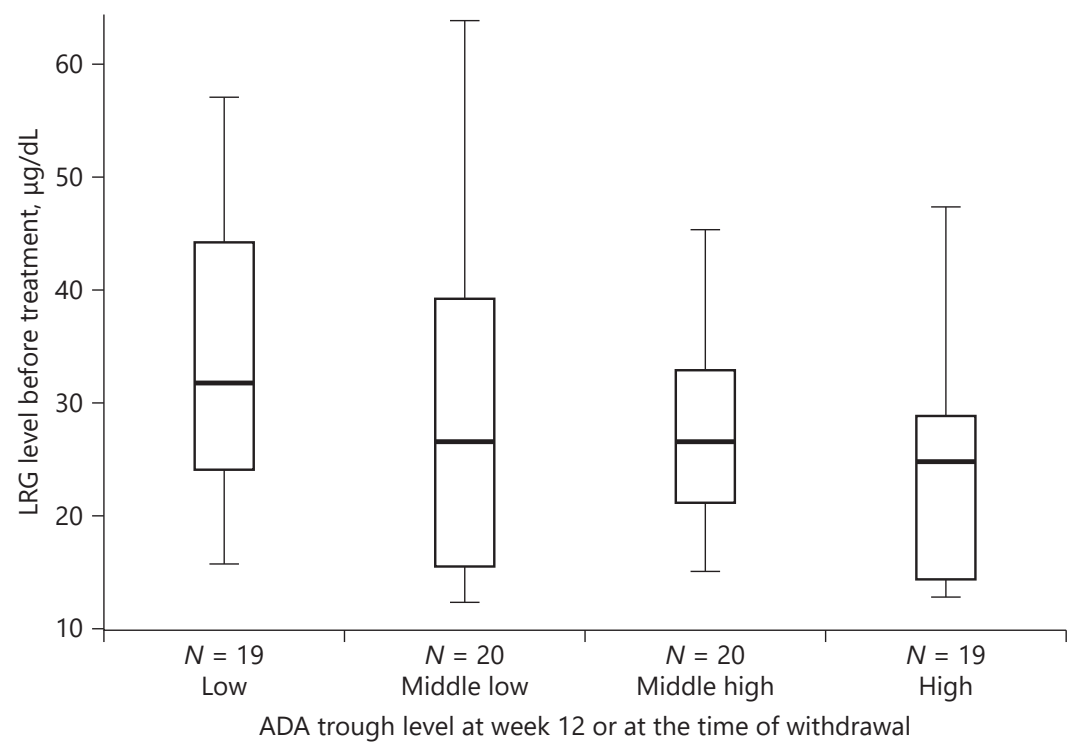

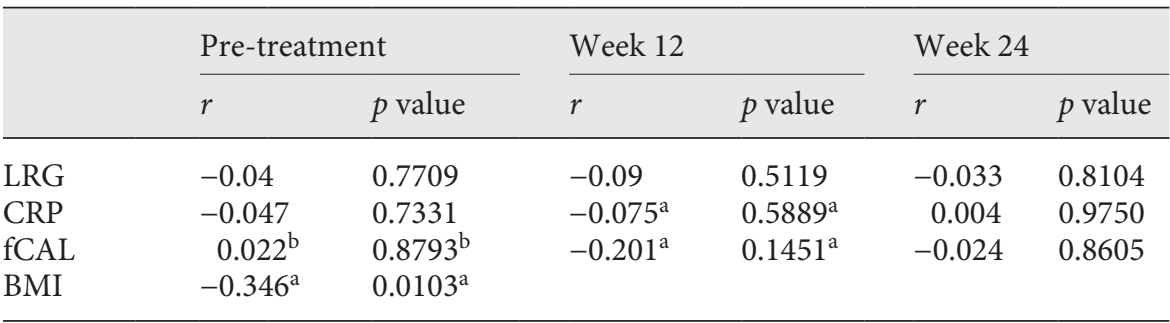

ADA, adalimumab; BMI, body mass index; CRP, C-reactive protein; fCAL; fecal calprotectin; LRG, leucine-rich alpha-2, glycoprotein. ${ }^{a}$ Data for one case are unavailable. ${ }^{\mathrm{b}}$ Data for 4 cases are unavailable.
Figure 4 shows a comparison of the pretreatment LRG level among quartiles classified by the ADA trough level at week 12 or at the time of withdrawal. The mean LRG level was the highest in patients belonging to the lowest quartile of ADA trough level $(32.2 \pm 12.5 \mu \mathrm{g} / \mathrm{mL}) \mathrm{com}$ pared with the other 3 groups $(27.4 \pm 15.8$ in the middlelow quartile, $26.4 \pm 8.7$ in the middle-high quartile, and $24.6 \pm 12.4 \mu \mathrm{g} / \mathrm{mL}$ in the highest quartile), suggesting that LRG may be particularly predictive of a low ADA trough level.

Table 3 summarizes the correlation between ADA trough levels at week 52 and biomarkers at weeks 0,12 , and 24. There was a statistically significant correlation between the ADA trough level at week 52 or withdrawal and pre-treatment BMI $(p=0.0103$ and $r=-0.346)$. However, the ADA trough level at week 52 after ADA treat- ment was not significantly associated with pretreatment $\mathrm{CRP}(p=0.7331$ and $r=-0.047)$, fCAL $(p=0.8793$ and $r$ $=0.022)$, or LRG $(p=0.7709$ and, $r=-0.04)$. Similarly, the ADA trough level at week 52 showed no significant association with CRP, fCAL, or LRG at weeks 12 and 24 .

\section{Discussion}

We attempted a sub-analysis of data obtained in a prospective, multicenter, observational study (PLANET) to investigate serial changes in a novel biomarker - LRG during ADA treatment for patients with active $\mathrm{CD}$ and UC. We found that LRG, rather than CRP or fCAL, was a marker for predicting the ADA trough level in patients with IBD treated with ADA. 
TDM has been suggested to play an important role not only for predicting patients at risk of treatment failure but also for the prediction of adequate response, aiming to achieve sustain remission [5]. Roblin et al. [6] reported that trough levels of ADA in the serum of patients without AAA were reliable predictors of a favorable clinical response. Mazor et al. [9] reported that ADA trough levels showed an inverse correlation with disease activity in CD. Nakase et al. [10] attempted a sub-analysis of data obtained by the DIAMOND study and reported that higher ADA trough levels and negative AAA status were significantly associated with clinical remission in CD patients who had been naive to biologics. Furthermore, Watanabe et al. [11] reported that higher ADA trough levels were associated with better endoscopic response and a higher rate of mucosal healing.

In our study, serum trough levels of ADA and AAA were measured at weeks 12 and 52, and 8 of 78 (10.2\%) patients were found to be AAA-positive. Also, the ADA trough level was significantly higher in AAA-negative patients than in AAA-positive patients. In a prospective clinical trial of ADA in patients with CD, Sandborn et al. [19] reported that 7 of 269 (2.6\%) patients were determined to be positive for AAA. Among those patients, 84 (31.2\%) had been receiving treatment with concomitant immunosuppressants [19]. While Wang et al. [20] reported that $44 \%$ of patients were positive for AAA, the positivity rate of AAA among Japanese $\mathrm{CD}$ patients naive to biologics in the DIAMOND study was only $8.6 \%$ [21]. The positivity rate of AAA among our patients in the PLANET study was similar to that found in DIAMOND, probably because $90 \%$ of our patients had been naive to biologics.

This study is the first prospective trial to compare LRG and the ADA trough level for patients with $\mathrm{CD}$ and UC. The ADA trough level at week 12 or at the time of ADA withdrawal was significantly and inversely associated with LRG at the time just prior to beginning ADA treatment. These results confirmed that LRG may be a biomarker for disease activity and for the prediction of TDM in patients receiving ADA treatment, while CRP or fCAL are not appropriate for TDM. The higher sensitivity of LRG as part of TDM may be explained by a wide distribution of LRG values among IBD patients [14].

CRP is a protein that is representative of acute-phase reactants. The liver, under the stimulation of circulating interleukin (IL)-6, is the primary organ for the production of CRP. In contrast, the primary site for the production of LRG has been suggested to be intestinal epithelial cells stimulated by IL- 6 , TNF-alpha, and IL-22, among

LRG, Adalimumab Trough Level, and Antidrug Antibodies other cytokines. It has also been reported that among those cytokines, TNF-alpha is the major one that drives LRG production $[22,23]$. Therefore, it seems likely that anti-TNF treatment can dramatically suppress LRG production and, thereby, reduce serum LRG levels. In this regard, our observation of a close association between LRG and ADA trough levels seems reasonable and clinically significant.

When we classified our subjects into quartiles according to the ADA trough level at week 12, the mean LRG value $(27.6 \mu \mathrm{g} / \mathrm{mL})$ at pretreatment was the highest in the group with the lowest ADA trough level. In contrast, the mean value of LRG was similar in the other 3 groups. While the practical cutoff value needs to be validated, we presume that pretreatment LRG may be applicable for the prediction of a low ADA trough level, and presumably poor response to $\mathrm{ADA}$, during the induction of remission in patients with IBD.

There was a significant correlation between the ADA trough level at week 52 and pre-treatment BMI. In contrast, pre-treatment biomarkers did not show any significant correlations with LRG. While the latter result may be a consequence of the small sample size at week 52 due to withdrawal from the study, the former result again suggested that BMI may be a significant factor associated with the ADA trough level during long-term treatment. In a sub-analysis of the DIAMOND study, Nakase et al. [10] reported an inverse correlation between body weight and ADA trough levels at week 12. In consideration of those prior reports and our results, BMI and fat content seem to be inevitable for the prediction of responses to ADA. Taken together, dose adjustment for ADA according to BMI may be considered to improve treatment efficacy.

There are several limitations to this study. First, because we did not measure the actual AAA titer, we were unable to make a predictive model for the presence of AAA. However, because the positivity rate of AAA and the practical ADA trough level were similar to those found in a prospective 52-week study [21], we believe that LRG is predictive of AAA titers. This needs to be further elucidated. Second, measurement of CRP was not centralized, but, rather, it was measured at each institution. Therefore, it seems possible that the value of CRP was heterogeneous, especially in patients with low CRP values. This may have negatively affected the evaluation of the predictive value of CRP. Third, lack of data of AAA and ADA trough levels in a proportion of our cohort and a small sample size, particularly of AAA-positive patients, suggest the need for a validation study. On the basis of the 
results of the present study, we are planning to establish a larger prospective cohort to examine associations between LRG and various biologics, including ADA.

\section{Conclusion}

We found that in patients with UC or CD treated by ADA, LRG, rather than CRP or fCAL, may be a marker for predicting the trough level of ADA and the presence of AAA as part of TDM of ADA. The clinical value of LRG as a TDM marker needs to be further clarified.

\section{Acknowledgments}

The authors are grateful to the following members of the PLANET study group: Hiroki Tanaka (Sapporo Welfare Hospital), Fuminao Takeshima (Nagasaki University), Shingo Kato (Saitama Medical University General Medical Center), Takehiro Torisu (Kyushu University), Yuki Ohta (Chiba University), Kenji Watanabe (Hyogo Medical College), Naoki Yoshimura (Tokyo Yamate Medical Center), Taku Kobayashi (Kitasato University Kitasato Institute Hospital), Akiko Shiotani (Kawasaki Medical College), Fumihito Hirai (Fukuoka University), Sakiko Hiraoka (Okayama University), Mamoru Watanabe (Tokyo Medical and Dental University), Minoru Matsuura (Kyorin University), Shohei Nishimoto (Eisai Co. Ltd), and Tetsuo Takehara (Osaka University).

\section{Statement of Ethics}

This study was registered in the University Hospital Medical Information Network (registration \#000019958), and it was conducted according to the Declaration of Helsinki and Ethical Guidelines for Medical and Health Research Involving Human Subjects provided by the Japan Ministry of Health, Labour and Welfare. All patients provided written informed consent.

\section{Conflict of Interest Statement}

S.S.: AbbVie, Tanabe Mitsubishi, and Janssen Pharmaceutical; K.M.: EA Pharma, Thermo Fisher Scientific, Sekisui Medical, and Alfresa Pharma; H.I.: AbbVie, EA Pharma, Eisai, Mitsubishi Tanabe Pharma, Mochida Pharmaceutical, Kyorin Pharmaceutical, Janssen, Zeria Pharmaceutical, Takeda Pharmaceutical, Sekisui Medical, AbbVie, Kissei, and Nippon Kayaku; T.K.: EA Pharma, Sekisui Medical, and Thermo Fisher Scientific; T.M.: Abbvie, Tanabe Mitsubishi, Janssen Pharmaceutical, EA Pharma, Nippon Kayaku, and Kyorin.

\section{Funding Sources}

This work was supported by Eisai Co., Ltd. Eisai Co., Ltd was involved in the study design but not in data acquisition or analysis.

\section{Author Contributions}

S.Y., S.S., K.M., S.M., H.I., T.N., T.K., and T.M. designed and conducted this study. S.Y., S.S., K.M., S.M., H.I., T.N., T.K., and T.M. collected data. S.Y., and T.M. interpreted the data. S.Y., and T.M. drafted the manuscript. All the authors confirmed and approved the manuscript.

\section{Data Availability Statement}

The data that support the findings of this study are not publicly available because there is no appropriate site for uploading at present. The data underlying this article will be shared on reasonable request to the corresponding author.

\section{References}

1 Harbord M, Eliakim R, Bettenworth D, Karmiris K, Katsanos K, Kopylov U, et al. European Crohn's and Colitis Organization. Third european evidence-based consensus on diagnosis and management of ulcerative colitis. Part 2: current management. J Crohns Colitis. 2017;11(7):769-84.

2 Sands BE, Feagan BG, Rutgeerts P, Colombel JF, Sandborn WJ, Sy R, et al. Effects of vedolizumab induction therapy for patients with Crohn's disease in whom tumor necrosis factor antagonist treatment failed. Gastroenterology. 2014;147(3):618-27.e3.

3 Feagan BG, Sandborn WJ, Gasink C, Jacobstein D, Lang Y, Friedman JR, et al. Ustekinumab as induction and maintenance therapy for Crohn's disease. N Engl J Med. 2016;375(20):1946-60.
4 Turner D, Ricciuto A, Lewis A, D’Amico F, Dhaliwal J, Griffiths AM, et al. STRIDE-II: an update on the selecting therapeutic targets in inflammatory bowel disease (STRIDE) initiative of the international organization for the study of IBD (IOIBD): determining therapeutic goals for treat-to-target strategies in IBD. Gastroenterology. 2021 Apr;160(5):1570-83.

5 Yarur AJ, Rubin DT. Therapeutic drug monitoring of anti-tumor necrosis factor agents in patients with inflammatory bowel diseases. Inflamm Bowel Dis. 2015;21(7):1709-18.

6 Roblin X, Rinaudo M, Del Tedesco E, Phelip JM, Genin C, Peyrin-Biroulet L, et al. Development of an algorithm incorporating pharmacokinetics of adalimumab in inflammatory bowel diseases. Am J Gastroenterol. 2014; 109(8):1250-6.
7 Afif W, Loftus EV Jr, Faubion WA, Kane SV, Bruining DH, Hanson KA, et al. Clinical utility of measuring infliximab and human antichimeric antibody concentrations in patients with inflammatory bowel disease. Am J Gastroenterol. 2010;105(5):1133-9.

8 Yanai H, Lichtenstein L, Assa A, Mazor Y, Weiss B, Levine A, et al. Levels of drug and antidrug antibodies are associated with outcome of interventions after loss of response to infliximab or adalimumab. Clin Gastroenterol Hepatol. 2015;13(3):522-30.e2.

9 Mazor Y, Almog R, Kopylov U, Ben Hur D, Blatt A, Dahan A, et al. Adalimumab drug and antibody levels as predictors of clinical and laboratory response in patients with Crohn's disease. Aliment Pharmacol Ther. 2014;40(6): $620-8$. 
10 Nakase $\mathrm{H}$, Motoya S, Matsumoto T, Watanabe K, Hisamatsu T, Yoshimura N, et al. Significance of measurement of serum trough level and anti-drug anitbody of adalimaumab as presonalised pharmacokinetics in patients with Crohn's disease. Aliment Pharmacol Ther. 2017;46(9):873-82.

11 Watanabe K, Matsumoto T, Hisamatsu T, Nakase H, Motoya S, Yoshimura N, et al. Clinical and pharmacokinetic factors associated with adalimumab-induced mucosal healing in patients with Crohn's disease. Clin Gastroenterol Hepatol. 2018;16(4):542-9.

12 Imaeda H, Bamba S, Takahashi K, Fujimoto T, Ban H, Tsujikawa T, et al. Relationship between serum infliximab trough levels and endoscopic activities in patients with Crohn's disease under scheduled maintenance treatment. J Gastroenterol. 2014;49(4):674-82.

13 Papamichael K, Cheifetz AS, Melmed GY, Irving PM, Vande Casteele N, Kozuch PL, et al. Appropriate therapeutic drug monitoring of biologic agents for patients with inflammatory bowel diseases. Clin Gastroenterol Hepatol. 2019;17(9):1655-68.e3.
14 Shinzaki S, Matsuoka K, Tanaka H, Takeshima F, Kato S, Torisu T, et al. Leucine-rich alpha-2 glycoprotein is a potential biomarker to monitor disease activity in inflammatory bowel disease receiving adalimumab: PLANET study. J Gastroenterol. 2021;56(6):560-9.

15 Matsuoka K, Kobayashi T, Ueno F, Matsui T, Hirai F, Inoue N, et al. Evidence-based clinical practice guidelines for inflammatory bowel disease. J Gastroenterol. 2018;53(3):305-53.

16 Schroeder KW, Tremaine WJ, Ilstrup DM. Coated oral 5-aminosalicylic acid therapy for mildly to moderately active ulcerative colitis. A randomized study. N Engl J Med. 1987; 317(26):1625-9.

17 Fujimoto M, Serada S, Suzuki K, Nishikawa A, Ogata A, Nanki T, et al. Leucine-rich a 2 -glycoprotein as a potential biomarker for joint inflammation during anti-interleukin- 6 biologic therapy in rheumatoid arthritis. Arthritis Rheumatol. 2015;67(8):2056-60.

18 Shankar G, Devanarayan V, Amaravadi L, Barrett YC, Bowsher R, Finco-Kent D, et al. Recommendations for the validation of immunoassays used for detection of host antibodies against biotechnology products. J Pharm Biomed Anal. 2008;48(5):1267-81.
19 Sandborn WJ, Hanauer SB, Rutgeerts P, Fedorak RN, Lukas M, Maclntosh DG, et al. Adalimumab for maintenance of Crohn's disease: results of the CLASSIC II trial. Gut. 2007;56(9):1232-9.

20 Wang SL, Hauenstein S, Ohrmund L, Shringarpure R, Salbato J, Reddy R, et al. Monitoring of adalimumab and antibodies-to-adalimumab levels in patient serum by the homogeneous mobility shift assay. J Pharm Biomed Anal. 2013;78-79:39-44.

21 Matsumoto T, Motoya S, Watanabe K, Hisamatsu T, Nakase H, Yoshimura N, et al. DIAMOND study group. Adalimumab monotherapy and a combination with azathioprine for Crohn's disease: a prospective, randomized trial. J Crohns Colitis. 2016;10(11):1259-66.

22 Serada S, Fujimoto M, Terabe F, Iijima H, Shinzaki S, Matsuzaki S, et al. Serum leucinerich alpha-2 glycoprotein is a disease activity biomarker in ulcerative colitis. Inflamm Bowel Dis. 2012;18(11):2169-79.

23 Shinzaki S, Matsuoka K, Iijima H, Mizuno S, Serada S, Fujimoto M, et al. Leucine-rich alpha-2 glycoprotein is a serum biomarker of mucosal healing in ulcerative colitis. J Crohns Colitis. 2017;11(1):84-91. 